\title{
Prediction of fat-free mass and percentage of body fat in neonates using bioelectrical impedance analysis and anthropometric measures: validation against the PEA POD
}

\author{
Barbara E. Lingwood ${ }^{1}$, Anne-Martine Storm van Leeuwen ${ }^{1,2}$, Angela E. Carberry ${ }^{1} \dagger$, Erin C. Fitzgerald ${ }^{1}$, \\ Leonie K. Callaway ${ }^{3}$, Paul B. Colditz ${ }^{1}$ and Leigh C. Ward ${ }^{4}$ \\ ${ }^{1}$ The University of Queensland, Perinatal Research Centre, UQ Centre for Clinical Research, Royal Brisbane and Women's \\ Hospital, Herston, QLD 4029, Australia \\ ${ }^{2}$ Department of Paediatrics, Nijmegen Medical Centre, Radboud University, Nijmegen, The Netherlands \\ ${ }^{3}$ School of Medicine, The University of Queensland, Brisbane, Australia \\ ${ }^{4}$ School of Chemistry and Molecular Biosciences, The University of Queensland, Brisbane, Australia \\ (Submitted 26 October 2010 - Final revision received 20 July 2011 - Accepted 20 July 2011 - First published online 15 September 2011)
}

\begin{abstract}
Accurate assessment of neonatal body composition is essential to studies investigating neonatal nutrition or developmental origins of obesity. Bioelectrical impedance analysis or bioimpedance analysis is inexpensive, non-invasive and portable, and is widely used in adults for the assessment of body composition. There are currently no prediction algorithms using bioimpedance analysis in neonates that have been directly validated against measurements of fat-free mass (FFM). The aim of the study was to evaluate the use of bioimpedance analysis for the estimation of FFM and percentage of body fat over the first 4 months of life in healthy infants born at term, and to compare these with estimations based on anthropometric measurements (weight and length) and with skinfolds. The present study was an observational study in seventy-seven infants. Body fat content of infants was assessed at birth, 6 weeks, 3 and 4.5 months of age by air displacement plethysmography, using the PEA POD body composition system. Bioimpedance analysis was performed at the same time and the data were used to develop and test prediction equations for FFM. The combination of weight + sex + length predicted FFM, with a bias of $<100 \mathrm{~g}$ and limits of agreement of $6-13 \%$. Before 3 months of age, bioimpedance analysis did not improve the prediction of FFM or body fat. At 3 and 4.5 months, the inclusion of impedance in prediction algorithms resulted in small improvements in prediction of FFM, reducing the bias to $<50 \mathrm{~g}$ and limits of agreement to $<9 \%$. Skinfold measurements performed poorly at all ages
\end{abstract}

Key words: Neonatal body composition assessment: Prediction equations: Bioelectrical impedance: Anthropometrics

Body composition in early life may play a key role in the programming of a variety of health outcomes, including hypertension, stroke, type 2 diabetes, obesity and $\mathrm{CVD}^{(1,2)}$. In this context, as well as in the assessment of feeding interventions for preterm infants, accurate assessment of neonatal body composition is essential.

A variety of methods have been used to assess body composition in infants (see Ellis $^{(3)}$ for a review) but all have some difficulties including lack of portability, lack of sensitivity, radiation exposure, requirement for the subject to be motionless, inability to perform repeat measurements and errors introduced by variable hydration of fat-free mass (FFM) in newborn infants. Air displacement plethysmography using an instrument called the PEA POD has been validated in healthy infants ${ }^{(4,5)}$, does not involve radiation exposure and does not require the infant to be restrained. Air displacement plethysmography is now considered a criterion method of body composition analysis in children ${ }^{(6)}$ and, in several studies, has provided more accurate measurements of body fat than dual-energy X-ray absorptiometry ${ }^{(7,8)}$. However, the instrument is expensive and bulky, and thus unlikely to be available outside major hospital facilities.

In contrast, bioelectrical impedance analysis instrumentation is inexpensive and portable, making it an attractive option for the assessment of infant body composition, particularly in smaller clinics and hospitals, or regional areas. Bioimpedance analysis provides reliable estimates of total body water in infants ${ }^{(9-12)}$ and is widely used for the assessment

Abbreviations: FFM, fat-free mass; IMP, impedance variable; $L$, length; $W$, weight; $S$, sex.

*Corresponding author: Dr B. E. Lingwood, fax +6173346 5594, email b.lingwood@uq.edu.au

† Present address: School of Public Health, The University of Sydney, Camperdown, NSW 2050 Australia. 
of FFM and body fat in adults ${ }^{(13-15)}$. However, Dung et al. ${ }^{(16)}$ reported that the use of impedance provides little benefit over weight alone for the estimation of FFM in preterm infants at approximately term-equivalent age. The estimation of FFM using impedance may be more reliable in healthy term infants who are more physiologically stable, but, to our knowledge, the use of bioimpedance analysis for the estimation of FFM and percentage of body fat has never been evaluated in healthy infants born at term.

The aim of the present study was to evaluate the use of bioimpedance analysis for the estimation of FFM and percentage of body fat over the first 4 months of life in healthy infants born at term, and to compare this with standard anthropometric measurements including weight and length and with skinfold measurements.

\section{Experimental methods}

Subjects were born at the Royal Brisbane and Women's Hospital, Brisbane, Australia, between April 2007 and April 2009. The study was conducted according to the guidelines laid down in the Declaration of Helsinki and all procedures involving human subjects were approved by the Human Research Ethics Committees of both the Royal Brisbane and Women's Hospital and the University of Queensland (Brisbane, Australia). Written informed consent was obtained from one parent of all subjects. All measurements were carried out at convenience to the subjects and the medical and nursing staff attending to the subjects.

Subjects considered eligible for the study were healthy term infants ranging in gestational age from 37 to 42 weeks born to women with a BMI between 18.5 and $25 \mathrm{~kg} / \mathrm{m}^{2}$ based on weight at the first antenatal visit. Infants were excluded if there was a history of maternal illness or gestational diabetes, infants were small-for-gestational age (below the 10th percentile for birth weight), from a multiple birth set, or were diagnosed with congenital abnormalities. A total of seventyseven infants were recruited to the study and measured at birth. Not all subjects attended for follow-up measurements and fifty-four infants were assessed at 6 weeks, fifty-five at 3 months and fifty-three at 4.5 months.

Body composition measurements were performed at birth $(0-4 \mathrm{~d}), 6$ weeks, 3 and 4.5 months of age using the PEA POD (Life Measurement, Inc., Concord, CA, USA) as described previously ${ }^{(17)}$. Infant length was measured heel to crown using an infant length board (Ellard Instrumentation Limited, Monroe, WA, USA).

\section{Bioelectrical impedance analysis}

Bioimpedance measurements were made using the Impedimed SFB7 (Impedimed, Brisbane, QLD, Australia), a single channel, tetra-polar bioimpedance spectroscopy device that measures resistance and reactance at 256 logarithmically spaced frequencies between 4 and $1000 \mathrm{kHz}$. Skin preparation and electrode placement were as described previously ${ }^{(18)}$. Infants were mostly awake and quiet. Insulating material was used to ensure no contact of the limbs with the body or each other.
Data were fitted to a Cole plot of resistance against reactance ${ }^{(19)}$, using ImpediMed software (Bioimp version 5.2.2.0; Impedimed, Brisbane, QLD, Australia) supplied with the instrument. The following impedance values were determined:

(1) Resistance at infinite frequency $\left(R_{\text {inf }}\right)$. At this frequency, the current path is not affected by the cell membranes and hence this parameter should provide the best prediction of total body water and therefore $\operatorname{FFM}^{(13,19)}$.

(2) Resistance at the characteristic frequency $\left(Z_{\mathrm{C}}\right)$. At this frequency, where reactance is maximal, the ratio of current flow through the extra- and intra-cellular paths is independent of the membrane capacitance. This parameter has also been used for the prediction of total body water $^{(13,19)}$.

(3) Resistance at zero frequency $\left(R_{0}\right)$. At this frequency, the current is not able to penetrate the cell membranes and hence this parameter should provide the best prediction of extracellular water volume ${ }^{(13,19)}$.

The measured value of resistance at $50 \mathrm{kHz}$ (the parameter most commonly used in single-frequency impedance measurements) was also recorded.

All measurements were initially analysed using the default software settings (frequency range $10-500 \mathrm{kHz}$; Td correction on; no data rejection limit). In addition, every measurement was analysed using a frequency range customised for that infant. This was achieved by visual examination of the raw data (Td correction off) and identification of the portion of the curve that most closely resembled a semicircle with a clear maximal reactance. The frequency range for analysis was then set to cover this portion of the curve. Further adjustment of the curves was accomplished by changing the upper and/or lower frequency limits to create the best fit to the semicircle. We aimed to achieve a standard error of the estimate $<1 \cdot 0$. Where a better curve fit was achieved, as judged by a smaller error, Td correction was applied.

\section{Skinfold thickness}

Triceps and subscapular skinfold thicknesses ( $\mathrm{mm}$ ) were measured in triplicate using a Harpenden skinfold caliper (Baty International, Burgess Hill, West Sussex, UK) and the mean value was obtained. Measurements were made by trained personnel and percentage of body fat was calculated using the following equations:

$$
\begin{gathered}
\text { Male } \% \text { fat }=1.21 \sum-0.008 \sum^{2}-1.7 \\
\text { Female } \% \text { fat }=1.33 \sum-0.013 \sum^{2}-2.5
\end{gathered}
$$

where $\Sigma=$ triceps + subscapular skinfolds $(\mathrm{mm})^{(20)}$.

\section{Data analysis}

Statistical analysis was performed using SPSS version 17 (SPSS, Inc., Chicago, IL, USA). Statistical significance was defined as a $P$ value $<0 \cdot 05$. Descriptive statistics are presented as means and standard deviations. Multiple regression analysis was 
used to assess the relationship between FFM and potential predictive variables including anthropometric and impedance variables and to develop prediction equations. Standardised regression coefficients were used to assess the contribution of each variable to the overall regression.

To develop and test prediction equations, the subject cohort was divided into two groups using a random number generator. The model development group included approximately two-thirds of the subjects and was used to develop prediction equations for FFM. In this group, multiple regression analysis was used to define the relationship between combinations of anthropometric and impedance variables and FFM. The validation group consisted of the remaining subjects. In these subjects, the developed models were used to predict FFM and, from that, percentage of body fat (based on a two-compartment model of body weight $=\mathrm{FFM}+$ fat $)$, providing an independent assessment of the performance of the equations. Bland \& Altman analysis ${ }^{(21)}$ was used to compare the prediction of FFM and percentage of body fat based on anthropometric and impedance variables with the measurement obtained from the PEA POD. The bias or mean difference between the methods was used to determine whether the predictions under- or overestimated the mean FFM and percentage of body fat and by how much. The limits of agreement were calculated to indicate the possible extent of the variation between the predicted value and the PEA POD measurement for any individual. The slope of the regression line was used to determine whether the bias was equal across the range of the measurements.

\section{Results}

The subject characteristics are described in Table 1. Of the evaluated infants, $89 \%$ had Caucasian ethnicity.

\section{Relationship between impedance variables and fat-free} mass

Impedance was negatively correlated with FFM at all ages but the strength of this correlation was low at birth, 6 weeks and 3 months of age (Fig. 1). The correlation was not consistently improved by customised fitting of the Cole plots. Where improvements were made, they were small and, in some cases, the strength of the correlation was reduced. Consequently, only the results of default analysis will be discussed. Significant correlations with FFM were found for $R_{50}, Z_{\mathrm{C}}$ and $R_{0}$ at birth and 3 months and for $R_{50}, Z_{\mathrm{C}}, R_{0}$ and $R_{\text {inf }}$ at 4.5 months.

Since impedance is proportional to the length $(L)$ and inversely proportional to the cross-sectional area $(A)$ of a conductor, and volume equals $L \times A$, it can be shown that the appropriate predictor for the volume of the conductor is the impedance quotient $L^{2} / \mathrm{IMP}$, where IMP is the impedance ${ }^{(13,22)}$. When the impedance quotient $L^{2} /$ IMP (where IMP is any impedance variable) was used in combination with weight and sex, this resulted in little or no improvement in the overall correlation compared with the use of weight + sex + length at birth and 6 weeks (Table 2). At these ages, the standardised regression coefficient for $L^{2} / \mathrm{IMP}$ was generally smaller than that for length and was never significant. At 3 and 4.5 months, substituting $L^{2} /$ IMP for length resulted in a small improvement in the overall correlation except when $L^{2} / R_{\text {inf }}$ was used at 4.5 months. At 3 months, the standardised regression coefficient was significant for $L^{2} / Z_{\mathrm{C}}$ and $L^{2} / R_{0}$, and for $L^{2} / R_{0}$, it was slightly greater than that for length. At 4.5 months, the coefficient for the quotients containing $R_{50}, Z_{\mathrm{C}}$ or $R_{0}$ was significant and was substantially greater than that for length alone. The coefficient for $L^{2} / R_{\text {inf }}$ was neither significant, nor greater than that for length at any age and the inclusion of this quotient produced little or no improvement in the overall correlation at any age.

\section{Prediction of fat-free mass}

There was no difference between the model development and validation groups in terms of birth weight, gestational age, or age, weight, length or FFM at any of the measurement times. Prediction of FFM based on weight + sex + length was accurate at all ages, with the bias generally $<100 \mathrm{~g}$ (Fig. 2). The mean bias was $1.5 \%$ of FFM at birth, $2.6 \%$ at 6 weeks, $0.6 \%$ at 3 months and $1.1 \%$ at 4.5 months. Limits of agreement were $200-500 \mathrm{~g}$, representing a possible error of $6-10 \%$ of

Table 1. Characteristics of the study subjects

(Mean values and standard deviations)

\begin{tabular}{|c|c|c|c|c|c|c|c|c|}
\hline & \multicolumn{2}{|c|}{ Birth $(n 77)$} & \multicolumn{2}{|c|}{6 weeks $(n 54)$} & \multicolumn{2}{|c|}{3 months ( $n 55)$} & \multicolumn{2}{|c|}{4.5 months ( $n 53)$} \\
\hline & Mean & SD & Mean & SD & Mean & SD & Mean & SD \\
\hline Birth weight $(\mathrm{kg})$ & 3.60 & 0.41 & & & & & & \\
\hline Gestational age (weeks) & $40 \cdot 0$ & $1 \cdot 1$ & & & & & & \\
\hline \multicolumn{9}{|l|}{$\operatorname{Sex}(n)$} \\
\hline Male & \multicolumn{2}{|c|}{41} & & & & & & \\
\hline Female & \multicolumn{2}{|c|}{36} & & & & & & \\
\hline Age at measurement (days or weeks) & $2 \cdot 1 \mathrm{~d}$ & $1.1 \mathrm{~d}$ & 6.3 weeks & 0.5 weeks & 12.7 weeks & 0.6 weeks & 19.4 weeks & 0.8 weeks \\
\hline Weight at measurement $(\mathrm{kg})^{\star}$ & 3.395 & 0.409 & 4.997 & 0.537 & 6.423 & 0.695 & $7 \cdot 163$ & 0.846 \\
\hline Length $(\mathrm{cm})$ & $51 \cdot 0$ & $2 \cdot 3$ & $57 \cdot 7$ & $2 \cdot 3$ & 61.9 & 2.5 & $65 \cdot 5$ & $2 \cdot 3$ \\
\hline FFM $(\mathrm{kg}) \dagger$ & 3.045 & 0.320 & 3.976 & 0.382 & 4.667 & 0.457 & $5 \cdot 201$ & 0.567 \\
\hline Body fat (\%)† & $10 \cdot 0$ & $4 \cdot 1$ & $20 \cdot 2$ & $4 \cdot 3$ & 24.9 & 4.4 & $26 \cdot 7$ & $6 \cdot 0$ \\
\hline
\end{tabular}

FFM, free-fat mass.

* Measured to the nearest $0.1 \mathrm{~g}$ using the PEA POD's integrated scale.

$\dagger$ Assessed by PEA POD as the reference method. 


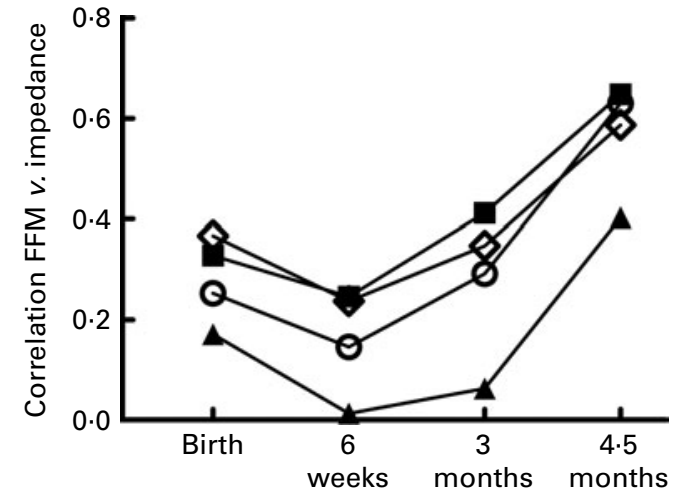

Fig. 1. Correlation coefficients for the relationship between impedance variables $\left(R_{\text {inf }}(-\leftarrow), Z_{\mathrm{C}}(-)\right), R_{0}(--)$ or $\left.R_{50}(-)\right)$ and fat-free mass (FFM) from birth to 4.5 months.

FFM at birth and $8-13 \%$ at later time points. Use of the impedance quotient $\left(L^{2} / \mathrm{IMP}\right)$ instead of length resulted in small increases in bias and limits of agreement at birth and 6 weeks of age. At 3 months, the use of impedance quotients containing $R_{50}, Z_{\mathrm{C}}$ or $R_{0}$ resulted in small increases in the bias. At 4.5 months, prediction of FFM using weight $+\mathrm{sex}+L^{2} / R_{0}$ resulted in decreased bias $(15 v .38 \mathrm{~g})$ and decreased limits of agreement ( -8.3 to $7.7 \% v$. -11.2 to $9.6 \%$ ) compared with prediction using weight + sex + length (Fig. 2). At birth, 3 and 4.5 months, the use of weight + sex + length overestimated FFM compared with the PEA POD at the low end of the range but underestimated it at the higher values. This did not occur at 3 and 4.5 months when weight + sex + $L^{2} / R_{0}$ was used.

\section{Prediction of percentage of body fat}

Prediction of the percentage of body fat using impedance requires first the prediction of FFM as described above and then calculation of the percentage of fat using FFM and body weight. Because fat is a smaller proportion of body weight than FFM, errors in the prediction of FFM are magnified by this calculation. When compared with PEA POD measurements, the bias for the prediction of percentage of body fat based on weight, length and sex at 3 and 4.5 months was small ( $<1 \%$ body fat). The bias was larger $(1 \cdot 2-1.9 \%$ body fat) at earlier ages. However, the limits of agreement were wide, indicating that this method of estimating the percentage of body fat in neonates may be as much as $9.5 \%$ fat below or $10.5 \%$ fat above the PEA POD measurement for an individual baby. This represents an

Table 2. Multiple regression analysis of weight $(W, \mathrm{~kg})$, sex $(\mathrm{S}+)$ and length $(L, \mathrm{~cm})$ or impedance quotients $\left(L^{2} /\right.$ impedance variable) for predicting fat-free mass (FFM)

\begin{tabular}{|c|c|c|c|c|c|}
\hline \multirow[b]{2}{*}{ Predictive variables } & \multirow[b]{2}{*}{ Overall $r$} & \multicolumn{3}{|c|}{ Standardised coefficients } & \multirow[b]{2}{*}{ Prediction equations for FFM } \\
\hline & & $W$ & $S$ & $L$ or $L^{2} / \mathrm{IMP}$ & \\
\hline \multicolumn{6}{|l|}{ Birth } \\
\hline W & 0.93 & $0.93^{\star *}$ & & & $0.578+0.727 W$ \\
\hline$W+S$ & 0.94 & $0.87^{\star \star}$ & $-0 \cdot 15^{\star \star}$ & & $0.864+0.682 W-0.093 S$ \\
\hline$W+S+L$ & 0.94 & $0.83^{\star \star}$ & $-0.14^{\star \star}$ & 0.07 & $0.507+0.646 W-0.089 S+0.009 L$ \\
\hline$W+S+L^{2} / R_{\mathrm{inf}}$ & 0.94 & $0.89^{\star \star}$ & $-0 \cdot 14^{\star \star}$ & -0.04 & $0.874+0.696 W-0.089 S+0.009 L / R_{\mathrm{inf}}$ \\
\hline$W+S+L^{2} / R_{50}$ & 0.94 & $0.86^{\star \star}$ & $-0.13^{\star \star}$ & 0.04 & $0.822+0.669 W-0.081 S+0.016 L / R_{50}$ \\
\hline$W+S+L^{2} / Z_{C}$ & 0.94 & $0.87^{\star \star}$ & $-0 \cdot 13^{\star \star}$ & 0.01 & $0.835+0.679 W-0.085 S+0.006 L / Z_{C}$ \\
\hline$W+S+L^{2} / R_{0}$ & 0.94 & $0.84^{\star \star}$ & $-0.11^{\star}$ & 0.07 & $0.779+0.655 W-0.072 S+0.037 L / R_{0}$ \\
\hline \multicolumn{6}{|l|}{6 weeks } \\
\hline w & 0.87 & $0.87^{\star *}$ & & & $0.901+0.6159 W$ \\
\hline$W+S$ & 0.89 & $0.81^{\star *}$ & $-0.20^{\star}$ & & $1.309+0.577 W-0.150 S$ \\
\hline$W+S+L$ & 0.89 & $0.74^{\star *}$ & $-0.16^{\star}$ & 0.13 & $0.260+0.528 W-0.125 S+0.022 L$ \\
\hline$W+S+L^{2} / R_{\mathrm{inf}}$ & 0.89 & $0.81^{* *}$ & $-0.19^{\star \star}$ & -0.06 & $1.322+0.588 W-0.148 S+0.009 L / R_{\text {inf }}$ \\
\hline$W+S+L^{2} / R_{50}$ & 0.89 & $0.81^{\star *}$ & $-0 \cdot 19^{\star}$ & 0.01 & $1.248+0.584 W-0.142 S+0.002 L / R_{50}$ \\
\hline$W+S+L^{2} / Z_{C}$ & 0.89 & $0.89^{\star \star}$ & $-0.19^{\star}$ & 0.00 & $1.253+0.585 W-0.143 S+0.001 L Z_{C}$ \\
\hline$W+S+L^{2} / R_{0}$ & 0.89 & $0.79^{\star \star}$ & $-0.17^{\star}$ & 0.06 & $1.169+0.568 W-0.128 S+0.032 L / R_{0}$ \\
\hline \multicolumn{6}{|l|}{3 months } \\
\hline$w$ & 0.84 & $0.84^{* *}$ & & & $1.210+0.554 W$ \\
\hline$W+S$ & 0.88 & $0.79^{\star \star}$ & $-0.25^{\star \star}$ & & $1.754+0.518 W-0.224 S$ \\
\hline$W+S+L$ & 0.89 & $0.66^{\star *}$ & $-0 \cdot 19^{\star \star}$ & $0.23^{\star *}$ & $-0.338+0.434 W-0.177 S+0.041 L$ \\
\hline$W+S+L^{2} / R_{\mathrm{inf}}$ & 0.90 & $0.79^{\star \star}$ & $-0 \cdot 24^{\star \star}$ & 0.07 & $1.533+0.531 W-0.223 S+0.017 L / R_{\text {inf }}$ \\
\hline$W+S+L^{2} / R_{50}$ & 0.90 & $0.74^{\star \star}$ & $-0 \cdot 21^{\star \star}$ & 0.15 & $1.458+0.498 W-0.197 S+0.067 L / R_{50}$ \\
\hline$W+S+L^{2} / Z_{C}$ & 0.91 & $0.73^{\star *}$ & $-0 \cdot 21^{\star \star}$ & $0 \cdot 18^{\star}$ & $1.383+0.487 W-0.192 S+0.081 L Z_{C}$ \\
\hline$W+S+L^{2} / R_{0}$ & 0.92 & $0.67^{\star \star}$ & $-0 \cdot 19^{\star \star}$ & $0.26^{* *}$ & $1.315+0.449 W-0.169 S+0.153 L / R_{0}$ \\
\hline \multicolumn{6}{|l|}{4.5 months } \\
\hline W & 0.74 & $0.74^{\star \star}$ & & & $1.491+0.521 W$ \\
\hline$W+S$ & 0.86 & $0.65^{\star \star}$ & $-0.43^{\star \star}$ & & $2.659+0.458 W-0.488 S$ \\
\hline$W+S+L$ & 0.87 & $0.57^{\star \star}$ & $-0.38^{\star \star}$ & $0 \cdot 17$ & $-0.044+0.397 W-0.427 S+0.045 L$ \\
\hline$W+S+L^{2} / R_{\mathrm{inf}}$ & 0.87 & $0.61^{* *}$ & $-0.40^{\star \star}$ & 0.15 & $2.484+0.416 W-0.430 S+0.040 L / R_{\mathrm{inf}}$ \\
\hline$W+S+L^{2} / R_{50}$ & 0.88 & $0.49^{\star \star}$ & $-0.34^{\star \star}$ & $0.30^{* *}$ & $2.203+0.334 W-0.361 S+0.185 L / R_{50}$ \\
\hline$W+S+L^{2} / Z_{C}$ & 0.89 & $0.46^{\star \star}$ & $-0 \cdot 30^{\star \star}$ & $0.35^{\star \star}$ & $2.059+0.313 W-0.320 S+0.201 L / Z_{C}$ \\
\hline$W+S+L^{2} / R_{0}$ & 0.89 & $0.41^{\star \star}$ & $-0 \cdot 26^{\star \star}$ & $0.41^{\star \star}$ & $1.909+0.280 W-0.279 S+0.305 L / R_{0}$ \\
\hline
\end{tabular}

${ }^{\star} P<0.05,{ }^{\star \star} P<0.01$ for statistically significant standardised regression coefficient $\dagger 1=$ male; $2=$ female. 

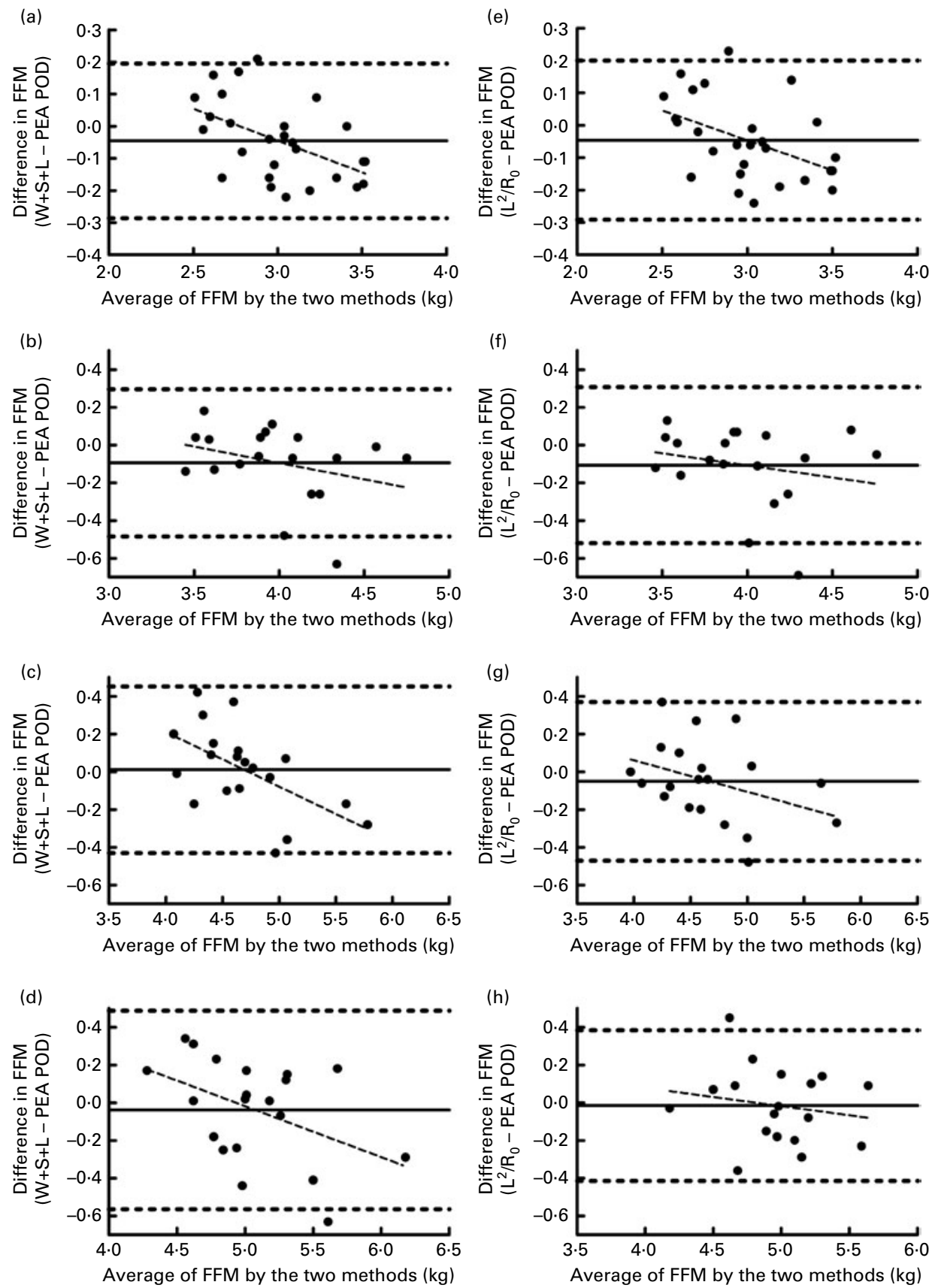

Fig. 2. Bland and Altman plots comparing the prediction of fat-free mass (FFM) by the PEA POD with (a-d) weight $(W)+\operatorname{sex}(S)+$ length $(L)$ or $(e-h)$ $W+S+L^{2} / R_{0}$ at $(\mathrm{a}, \mathrm{e})$ birth, (b, f) 6 weeks, (c, g) 3 months and (d, h) 4.5 months after birth. Bias is indicated by the solid horizontal line and limits of agreement (mean bias $( \pm 2 \mathrm{SD})$ ) by the dotted lines. The broken line represents the linear regression of the data. The slope of this line was not significantly different from zero for $W+S+L$ at 6 weeks, and for $W+S+L^{2} / R_{0}$ at 6 weeks, 3 months and 4.5 months.

error of up to $80 \%$ at birth, $28-45 \%$ at 6 weeks and $22-23 \%$ at 4.5 months.

Substituting the impedance quotient $\left(L^{2} / \mathrm{IMP}\right)$ for length in the prediction equation at birth made little difference to the bias or limits of agreement, irrespective of which impedance quotient was used. The best-performing prediction equation included weight, sex and $L^{2} / R_{0}$ at 4.5 months. The bias was very small and similar to that for weight, sex and length. The limits of agreement were lower, reducing the possible error to $6 \%$ fat above or below the PEA POD measurement representing an error of $19-21 \%$.

The estimation of percentage of body fat obtained from skinfold thickness measurements was also compared with PEA POD measurements in the validation group (Fig. 3). For this method, the bias was large and, from 6 weeks onwards, underestimated percentage of fat by 2.4-8.9 percentage points. At 4.5 months, the underestimation of the percentage of body fat was greater in infants with the highest body fat 

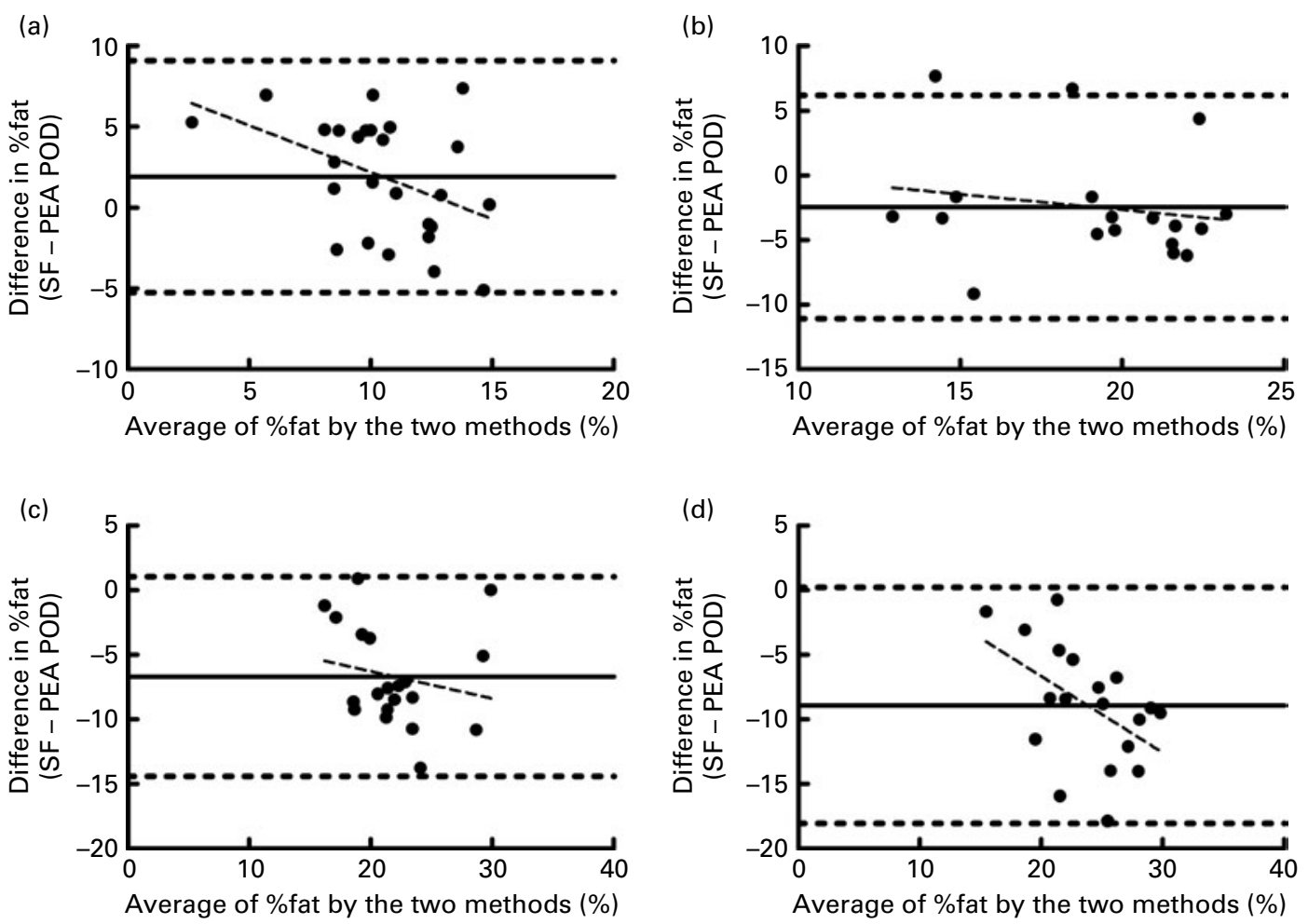

Fig. 3. Bland and Altman plots comparing the prediction of percentage of body fat by the PEA POD with skinfolds (SF) at (a) birth, (b) 6 weeks, (c) 3 months and (d) 4.5 months after birth. Bias is indicated by the solid horizontal line and limits of agreement (mean bias ( \pm 2 SD)) by the dotted lines. The broken line represents the linear regression of the data. The slope of this line was not significantly different from zero at 6 weeks and 3 months.

The limits of agreement were once again wide, with possible error ranging from $18 \%$ fat below to $9 \%$ fat above the PEA POD measurement.

\section{Discussion}

The use of bioimpedance analysis did not improve the prediction of FFM or percentage of body fat over that obtained from the combination of weight, sex and length, at birth or 6 weeks of age. Small improvements in the prediction of FFM and percentage of fat were found using $L^{2} / Z_{\mathrm{C}}$ or $L^{2} / R_{0}$ at 3 months or $L^{2} / Z_{\mathrm{C}}$ at 4.5 months. A larger improvement was found using $L^{2} / R_{0}$ at 4.5 months. This finding is consistent with the observation that the correlation between impedance and FFM is not strong, especially at younger ages. The steadily increasing correlation of impedance with FFM as age increases also suggests that the use of impedance would be more beneficial at older ages. Although the possible error in individual measurements is quite large, the small bias means that this method is suitable for comparison between groups.

The present findings are consistent with other studies that have shown that impedance provides little or no benefit over weight alone for the estimation of FFM or total body water in preterm infants ${ }^{(16)}$, and when used alone often provides an estimate of total body water which is greater than the weight ${ }^{(10)}$. In contrast, Tang et al. ${ }^{(12)}$ found some improvement in the prediction of total body water when impedance was included with weight in a group of sick infants including both term and preterm infants aged $1-7 \mathrm{~d}$. However, the improvement was extremely small ${ }^{(12)}$.

The use of bioimpedance analysis to estimate FFM is based on the assumption that fat is essentially non-conductive. However, adipose tissue has a wide range of conductivity which is dependent on the degree of perfusion ${ }^{(23)}$. In the embryo, there is a rich vascular supply to the adipose tissue, which becomes more sparse with growth and development ${ }^{(24)}$. In addition, the composition of fat in the neonate is different from that in the adult. The percentage of water is higher and quite variable, diminishing from a mean of $45-48 \%$ at birth to $27-38 \%$ at $6-10$ months and $20-26 \%$ in the adult ${ }^{(25)}$. The increased vascularisation of the adipose tissue and the high water content of fat in the early months of life may increase the conductivity of fat. Thus, the assumption that fat is not conductive may be incorrect and may invalidate the simple electrical model on which the Cole model is based. This may account for the poor correlation of impedance with FFM at the early ages, and the increasing correlation with age. The frequent and highly variable feeding patterns of infants, along with the rapidly changing hydration, make it difficult to standardise hydration states, and this may also contribute to variability in the measurements and the poor predictive performance of impedance.

The best correlation with FFM was found with the impedance variable $R_{0}$, a finding that is contrary to theoretical principles. Theoretically, $R_{\text {inf }}$ should provide the best prediction of total body water and FFM. However, we have noted, in the neonate, that the high-frequency end of the Cole plot is 
highly variable, making accurate fitting of the curve difficult in this region. This variability may be the result of difficulty in achieving 'ideal' conditions for measurement in the neonate ${ }^{(26,27)}$. Neonatal patients do not cooperate as readily as adults, and lack of movement is difficult to achieve and does affect the measurements ${ }^{(26)}$. There are also fewer data points (the frequencies of measurement are logarithmically spaced) at the high-frequency end of the curve. Individualising the fitting parameters for each baby did not improve the ability to predict FFM. In contrast, the low-frequency end of the curve is more predictable and contains more data points, making fitting of the curve more reliable and decreasing the variation in the estimation of $R_{0}$. This may account for the better prediction obtained with $R_{0} . R_{0}$ provides an estimate of extracellular water volume, which in a healthy adult is a consistent fraction of FFM, and thus would provide a good estimate of FFM. However, the ratio of extracellular to total body water in the neonate is highly variable, especially in the first few days of life, further compounding the difficulties of predicting FFM at this age. $Z_{\mathrm{C}}$ is also used to predict total body water or FFM in the adult ${ }^{(14)}$, and does provide a better estimate than $R_{\text {inf }}$ in the neonate, although this will still be affected by difficulties with fitting the curve at the highfrequency end of the spectrum. $R_{50}$ is the parameter most commonly used in single-frequency impedance measurements but provides no improvement over anthropometric measures when predicting FFM in the neonate.

Measurements of skinfold thickness likewise did not improve the prediction of percentage of body fat at any age and predicted percentage of fat less accurately than weight + sex + length at all ages. Skinfold measurements also correlate poorly with percentage of fat determined from the estimation of FFM based on total body water ${ }^{(28)}$. There are limited models available for the estimation of body fat in neonates using skinfold thickness. A theoretical model proposed by Dauncey et $a l .{ }^{(29)}$ requires nine measurements of body dimensions in addition to skinfold measurements but, to our knowledge, has not been validated. The equations used for the present study were based on equations for pre-pubescent males and females ${ }^{(20)}$ and utilised subscapular and triceps measurements. Better prediction for percentage of fat may have been obtained using the flank skinfold and the equation of Catalano et $a l .{ }^{(30)}$ that was derived specifically for neonates. Regardless of the model used, skinfold measurement requires extensive training and meticulous care to ensure accuracy and reproducibility between observers ${ }^{(30)}$.

A major strength of the present study is the use of a reference standard that measures percentage of fat and FFM. When FFM is derived from estimations of total body water as in many other studies, additional error is introduced due to uncertainty regarding the hydration constant used for the conversion of total body water to FFM, which may be very variable particularly in the first days of life. This is the first study to directly calibrate impedance against FFM in healthy term neonates. We are not aware of any other study that has evaluated the use of bioimpedance analysis for the prediction of percentage of fat in neonates. The PEA POD uses air displacement plethysmography to assess body composition in infants and has been validated in a number of studies $^{(4,5,31,32)}$. Although relatively new, air displacement plethysmography is already considered a criterion method for body composition in children ${ }^{(6)}$, and has been shown to provide better estimates than dual-energy X-ray absorptiometry in some studies ${ }^{(7,8)}$. The PEA POD takes into account the fact that hydration status is different in the neonate from that in the adult, and, in particular, allows for the greater hydration of FFM in the first week of life. Thus, it is the ideal instrument for the assessment of body composition in neonates, and for use as a reference standard when assessing other methods.

In conclusion, bioimpedance analysis does not provide any advantage over the use of simple anthropometric measures to estimate FFM and percentage of body fat in neonates at least until 3 months of age. At 3-4.5 months of age, bioimpedance analysis provides small improvements in the prediction of FFM but the prediction of percentage of body fat, although better than skinfold measurements, may still contain substantial error.

\section{Acknowledgements}

This study was supported by the Royal Brisbane and Women's Hospital Research Foundation. None of the authors has any conflict of interest. The authors' contributions were as follows: B. E. L. was primarily responsible for the concept and design, data analysis and interpretation, and drafting the manuscript; A.-M. S. V. L. assisted with the design, data acquisition and analysis, and drafting the manuscript; A. E. C. and E. C. F. were involved in the data acquisition and analysis, and critical revision of the manuscript; L. K. C. and P. B. C. were responsible for the study design and critical revision of the manuscript; L. C. W. contributed to the design, data analysis and interpretation, statistics and critical revision of the manuscript.

\section{References}

1. Wells JC, Chomtho S \& Fewtrell MS (2007) Programming of body composition by early growth and nutrition. Proc Nutr Soc 66, 423-434.

2. Chomtho S, Wells JC, Williams JE, et al. (2008) Infant growth and later body composition: evidence from the 4-component model. Am J Clin Nutr 87, 1776-1784.

3. Ellis KJ (2007) Evaluation of body composition in neonates and infants. Semin Fetal Neonatal Med 12, 87-91.

4. Ellis KJ, Yao M, Shypailo RJ, et al. (2007) Body-composition assessment in infancy: air-displacement plethysmography compared with a reference 4-compartment model. Am J Clin Nutr 85, 90-95.

5. Ma G, Yao M, Liu Y, et al. (2004) Validation of a new pediatric air-displacement plethysmograph for assessing body composition in infants. Am J Clin Nutr 79, 653-660.

6. Bosy-Westphal A, Danielzik S, Becker C, et al. (2005) Need for optimal body composition data analysis using airdisplacement plethysmography in children and adolescents. J Nutr 135, 2257-2262.

7. Fields DA \& Goran MI (2000) Body composition techniques and the four-compartment model in children. J Appl Physiol 89, 613-620. 
8. Gately PJ, Radley D, Cooke CB, et al. (2003) Comparison of body composition methods in overweight and obese children. J Appl Physiol 95, 2039-2046.

9. Ferreira DM \& Souza MN (2004) Bioelectrical impedance spectroscopy for the assessment of body fluid volumes of term neonates. Braz J Med Biol Res 37, 1595-1606.

10. Mayfield SR, Uauy R \& Waidelich D (1991) Body composition of low-birth-weight infants determined by using bioelectrical resistance and reactance. Am J Clin Nutr 54, 296-303.

11. Raghavan CV, Super DM, Chatburn RL, et al. (1998) Estimation of total body water in very-low-birth-weight infants by using anthropometry with and without bioelectrical impedance and H2[(18)O]. Am J Clin Nutr 68, 668-674.

12. Tang W, Ridout D \& Modi N (1997) Assessment of total body water using bioelectrical impedance analysis in neonates receiving intensive care. Arch Dis Child Fetal Neonatal Ed 77, F123-F126.

13. Chumlea WC \& Guo SS (1994) Bioelectrical impedance and body composition: present status and future directions. Nutr Rev 52, 1231-1231.

14. Cornish BH, Ward LC, Thomas BJ, et al. (1996) Evaluation of multiple frequency bioelectrical impedance and Cole-Cole analysis for the assessment of body water volumes in healthy humans. Eur J Clin Nutr 50, 159-164.

15. Lukaski HC, Bolonchuk WW, Hall CB, et al. (1986) Validation of tetrapolar bioelectrical impedance method to assess human body composition. J Appl Physiol 60, 1327-1332.

16. Dung NQ, Fusch G, Armbrust S, et al. (2007) Body composition of preterm infants measured during the first months of life: bioelectrical impedance provides insignificant additional information compared to anthropometry alone. Eur J Pediatr 166, 215-222.

17. Carberry AE, Colditz PB \& Lingwood BE (2010) Body composition from birth to 4.5 months in infants born to non-obese women. Pediatr Res 68, 84-88.

18. Lingwood BE, Coghlan JP, Ward LC, et al. (2000) Measurement of extracellular fluid volume in the neonate using multiple frequency bio-impedance analysis. Physiol Meas 21, 251-262.
19. Thomas BJ, Cornish BH \& Ward LC (1992) Bioelectrical impedance analysis for measurement of body fluid volumes: a review. J Clin Eng 17, 505-510.

20. Slaughter MH, Lohman TG, Boileau RA, et al. (1988) Skinfold equations for estimation of body fatness in children and youth. Hum Biol 60, 709-723.

21. Bland JM \& Altman DG (1986) Statistical methods for assessing agreement between two methods of clinical measurement. Lancet 1, 307-310.

22. Guo SS, Chumlea WC \& Cockram DB (1996) Use of statistical methods to estimate body composition. Am J Clin Nutr 64, (Suppl. 3), 428S-435S.

23. Grimnes S \& Martinsen $\varnothing \mathrm{G}$ (2008) Bioimpedance and Bioelectricity Basics, 2nd ed. London: Elsevier Ltd.

24. Ryan TJ (1992) Development of the cutaneous circulation. In Fetal and Neonatal Physiology, pp. 562-563 [RA Polin and WW Fox, editors]. Philadelphia, PA: W.B. Saunders Company.

25. Baker GL (1969) Human adipose tissue composition and age. Am J Clin Nutr 22, 829-835.

26. Sesmero MA, Mazariegos M, Pedron C, et al. (2005) Bioimpedance electrical spectroscopy in the first six months of life: some methodologic considerations. Nutrition 21, 567-573.

27. National Institutes of Health (1996) Bioelectrical impedance analysis in body composition measurement: National Institutes of Health Technology Assessment Conference Statement. Am J Clin Nutr 64, Suppl. 3, 524S-532S.

28. Davies PS \& Lucas A (1990) The prediction of total body fatness in early infancy. Early Hum Dev 21, 193-198.

29. Dauncey MJ, Gandy G \& Gairdner D (1977) Assessment of total body fat in infancy from skinfold thickness measurements. Arch Dis Child 52, 223-227.

30. Catalano PM, Thomas AJ, Avallone DA, et al. (1995) Anthropometric estimation of neonatal body composition. Am J Obstet Gynecol 173, 1176-1181.

31. Sainz RD \& Urlando A (2003) Evaluation of a new pediatric air-displacement plethysmograph for body-composition assessment by means of chemical analysis of bovine tissue phantoms. Am J Clin Nutr 77, 364-370.

32. Urlando A, Dempster P \& Aitkens S (2003) A new air displacement plethysmograph for the measurement of body composition in infants. Pediatr Res 53, 486-492. 